\title{
Comparação da disfunção do assoalho pélvico com função sexual e qualidade de vida em sobreviventes ao câncer ginecológico
}

\author{
Francielle Conceiçáo Nascimento (D), Julia Deitos (D), Clarissa Medeiros da Luz (C) \\ Universidade do Estado de Santa Catarina - UDESC, Florianópolis, SC, Brasil.
}

\begin{abstract}
Resumo: Objetivo: Comparar a contração objetiva do assoalho pélvico, estenose vaginal e dispareunia com a função sexual e a qualidade de vida relacionada à saúde de sobreviventes ao câncer ginecológico. Método: Estudo transversal com mulheres com diagnóstico de câncer ginecológico e com no mínimo seis meses de término do tratamento oncológico. A avaliação consistiu na aplicação da ficha de identificação, questionários (QS-F e FACT-G) e exame físico ginecológico. Resultados: Foram avaliadas 64 pacientes com média de idade de 50 anos, das quais $56,3 \%$ eram ativas sexualmente. $\mathrm{O}$ tipo de câncer mais frequente foi o de colo uterino $(62,5 \%)$, sendo o principal tratamento realizado a histerectomia $(54,7 \%)$. Observou-se a presença de dispareunia em $32,8 \%$ das mulheres ativas sexualmente, a estenose vaginal foi identificada em $45,3 \%$ dos casos, e 45,3\% apresentaram contração objetiva ausente do assoalho pélvico. A comparação das médias dos escores do questionário QS-F evidenciou diferença significante considerando a presença ou não de estenose vaginal e dispareunia, enquanto que a presença ou ausência da contração objetiva do assoalho pélvico não apresentou diferença significante na função sexual. Verificou-se associação entre estenose vaginal e dispareunia, porém a atividade sexual e a contração do assoalho pélvico não estiveram associadas à estenose vaginal. Além disso, a estenose vaginal, dispareunia e a contração do assoalho pélvico não apresentaram associação significante com a qualidade de vida. Conclusão: A presença de disfunção do assoalho pélvico nas sobreviventes ao câncer ginecológico indicou prejuízo na função sexual, sem impactar negativamente na qualidade de vida relacionada à saúde.
\end{abstract}

Palavras-chave: Assoalho Pélvico, Disfunção Sexual, Estenose Vaginal.

\section{Comparison of pelvic floor dysfunction with sexual function and quality of life in gynecological cancer survivors}

\begin{abstract}
Objective: To compare objective contraction of the pelvic floor, vaginal stenosis and dyspareunia with sexual function and health related quality of life in gynecological cancer survivors. Method: Cross-sectional study with women referred to oncological treatment for gynecological cancer with at least six months of the treatment. Study participants completed an initial evaluation form regarding demographic characteristics, and medical history, QS-F and FACT-G questionnaires, and gynecological physical examination. Results: Sixty-four patients with a mean age of 50 years, of which $56.3 \%$ were sexually active, were evaluated. The most frequent type of cancer was cervical cancer $(62.5 \%)$ and the main treatment was hysterectomy (54.7\%). The presence of dyspareunia was observed in $32.8 \%$ of sexually active women, vaginal stenosis was identified in $45.3 \%$ of the cases, and $45.3 \%$ presented no objective contraction of the pelvic floor. The comparison of QS-F questionnaire scores showed a significant difference considering the presence or absence of vaginal stenosis and dyspareunia, while the presence or absence of objective contraction of the pelvic floor did not show a significant difference in sexual function. There was an association
\end{abstract}

Autor para correspondência: Clarissa Medeiros da Luz, Programa de Pós-graduação em Fisioterapia, Centro de Ciências da Saúde e do Esporte, Universidade do Estado de Santa Catarina, Rua Pascoal Simone, 358, Coqueiros, CEP 88080-350, Florianópolis, SC, Brasil, e-mail: clarissa.medeiros@udesc.br

Recebido em Fev. 1, 2018; 1ª Revisão em Set. 11, 2018; Aceito em Mar. 20, 2019. 
between vaginal stenosis and dyspareunia, but sexual activity and contraction of the pelvic floor were not related to vaginal stenosis. In addition, vaginal stenosis, dyspareunia and contraction of the pelvic floor were not significantly associated with health-related quality of life. Conclusion: The presence of pelvic floor dysfunction in gynecological cancer survivors indicated impairment in sexual function with no negative impact on health-related quality of life.

Keywords: Pelvic Floor, Sexual Dysfunction, Vaginal Stenosis.

\section{Introdução}

O câncer ginecológico inclui as neoplasias malignas do corpo e colo uterino, ovários, endométrio, vagina ou vulva (RUTLEDGE et al., 2010) e é considerado um dos grupos de neoplasias mais comumente encontrados na população feminina (VAZ et al., 2011a), sendo esperados cerca de 29.440 novos casos para o ano de 2017 no Brasil (INSTITUTO..., 2015).

O tratamento do câncer ginecológico depende do estadiamento da doença, podendo incluir a cirurgia, radioterapia, quimioterapia, hormonioterapia e terapia-alvo ou a combinação dessas modalidades (RUTLEDGE et al., 2014). Apesar das terapias proporcionarem maior sobrevida, muitas vezes geram alteraçôes na anatomia e fisiologia pélvica, com destaque para a redução da função dos músculos do assoalho pélvico que pode refletir em incontinência ou retenção urinária e fecal, prolapsos genitais e disfunçôes sexuais (YANG et al., 2012; RUTLEDGE et al., 2014).

As disfunçôes do assoalho pélvico são tópicos importantes abordados nas complicaçôes pós-tratamento do câncer ginecológico (YE et al., 2014), sendo as queixas mais relatadas pelas mulheres o baixo desejo sexual, secura e alteraçóes anatômicas da região vaginal, anorgasmia e dispareunia (BRADFORD et al., 2015). Ao exame objetivo, a disfunção mais frequente é a estenose vaginal (YOSHIDA et al., 2015), relacionada à diminuição da largura e elasticidade da mucosa vaginal, provocando dor e desconforto durante a atividade sexual (CARTER et al., 2017) e dificultando a realizaçáo de exames ginecológicos de rotina, indispensáveis no seguimento clínico dessas mulheres (SILVA et al., 2010; ROS; ESPUNA, 2013).

As sobreviventes ao câncer ginecológico que sofrem com a presença dessas disfunçóes podem apresentar, ainda, um impacto psicossocial (RUTLEDGE et al., 2014) que afeta negativamente a qualidade de vida nos domínios de bem-estares físico, emocional, profissional e social (YANG et al., 2012; WESTIN et al., 2016). Observa-se que, as disfunções dos órgãos genitais nessas mulheres provocam sentimento de constrangimento ao compartilhar seus sintomas com familiares, amigos ou profissionais de saúde, ameaçando a imagem corporal, levando a uma baixa autoestima, perda da feminilidade e comprometimento da sexualidade (REIS; BEJI; COSKUN, 2010).

Alguns estudos sugerem que mulheres com disfunçóes do assoalho pélvico como dispareunia, atrofia vaginal (VAZ et al., 2011b; ZHOU et al., 2016; HUFFMAN et al., 2016; SEKSE; HUFTHAMMER; VIKA, 2017) e insatisfação sexual (BRETSCHNEIDER et al., 2017), apresentaram redução da qualidade de vida. A abordagem das disfunçôes do assoalho pélvico nas sobreviventes ao câncer ginecológico é cada vez mais frequente, entretanto, não há um padrão de tratamento indicado (BOBER et al., 2018), sendo necessário conhecer o real impacto destas disfunçóes na função sexual para um melhor direcionamento das intervençôes terapêuticas (WHICKER et al., 2017).

Diante do exposto, o presente estudo teve como objetivo comparar a funçáo do assoalho pélvico, estenose vaginal e dispareunia com a função sexual e a qualidade de vida relacionada à saúde de sobreviventes ao câncer ginecológico.

\section{Método}

Trata-se de um estudo transversal com mulheres admitidas na Maternidade Carmela Dutra, Florianópolis - SC, entre os anos de 2009 e 2013 para diagnóstico e/ou tratamento de câncer ginecológico de acordo com o Registro Hospitalar de Câncer (RHC) da instituição. A amostra foi não probabilística, de modo que foram recrutadas por meio de contato telefônico e convidadas a participar da pesquisa mulheres maiores de 18 anos com diagnóstico de câncer ginecológico (colo de útero, endométrio, ovários, vulva e vagina) classificado como tumor primário, residentes na Grande Florianópolis e com no mínimo seis meses de conclusão do tratamento oncológico. Foram excluídas aquelas que apresentassem recidiva tumoral ou metástase à distância diagnosticadas até o momento da coleta.

Para caracterização da amostra foi utilizada ficha de identificação contendo dados sociodemográficos, antecedentes ginecológicos e obstétricos, história da doença, tratamentos realizados e hábitos de vida. A estatura e a massa corporal foram avaliadas, 
respectivamente, por um estadiômetro (Sanny) e uma balança BC-558 Ironman Segmental Body Composition Monitor (Tanita), previamente calibrada. Foram verificadas as medidas com a participante descalça, na posição ereta e cabeça alinhada. O índice de massa corporal - IMC $\left(\mathrm{kg} / \mathrm{m}^{2}\right)$ foi calculado para posterior avaliaçáo do estado nutricional. Como pontos de corte foram adotados os recomendados pela Organização Mundial da Saúde (WORLD..., 1995).

A função sexual foi avaliada por meio do questionário Quociente Sexual - Feminino (QS-F) (ABDO, 2006). A presença de disfunção sexual foi considerada em escores abaixo de 60 pontos, que é o ponto de corte estabelecido para este fim no questionário QS-F (ABDO, 2006).

A qualidade de vida foi avaliada através do questionário Functional Assessment of Cancer Therapy - General (FACT-G). O escore total do instrumento resulta da soma das pontuações dos domínios e varia de 0 a 108 (WEBSTER; CELLA; YOST, 2003). Os valores mais elevados representam melhor qualidade de vida relacionada à saúde. Todos os questionários foram traduzidos e validados para o português, e autorizados para uso pelo grupo Facit (2015).

A avaliação da função muscular do assoalho pélvico foi realizada através da mensuração do grau de contração muscular pela mesma examinadora em todas as participantes através de toque bidigital, e classificada em diferentes graus funcionais, por meio da Avaliação Funcional do Assoalho Pélvico (AFA) proposta por Ortiz et al. (1994). No presente estudo, foram considerados os graus 0 e 1 como contração objetiva ausente e os graus 2, 3 e 4 como contração objetiva presente.

A estenose vaginal foi avaliada através de um conjunto de dilatadores vaginais de silicone (PURE Romance ${ }^{\circledR}$ ) composto por seis unidades com comprimentos e diâmetros variados, revestidos por preservativo tipo hospitalar e gel lubrificante. O grau de dilataçáo e comprimento vaginal foram definidos com o dilatador que permitisse a inserção completa no canal vaginal, sem que ocorresse a expulsão do instrumento. A estenose vaginal foi considerada presente para os dilatadores de número 1 e 2 ou nos casos em que não foi possível a introdução de nenhum tamanho (FLAY; MATTHEWS, 1995).

Os dados foram tabulados e analisados no pacote estatístico The Statistical Package for the Social Sciences $^{\circledR}$ (SPSS v.20.0). A caracterização da amostra foi apresentada na forma de estatística descritiva. Para verificar a distribuição dos dados foi utilizado o teste de Kolmogorov-Smirnov ou Shapiro-Wilk. A variável qualidade de vida foi descrita por meio da média, sendo que para fins de análise estatística, foram utilizadas as medianas dos escores categorizando-a em acima e abaixo. As médias dos escores do questionário QS-F e FACT-G foram comparadas considerando a presença ou ausência de estenose vaginal, dispareunia e contração objetiva do assoalho pélvico, utilizando o teste $t$ independente ou $U$ de Mann-Whitney. A associação entre as variáveis contração objetiva do assoalho pélvico, estenose vaginal, dispareunia, atividade sexual e qualidade de vida foi tratada através do teste Qui Quadrado ou Exato de Fisher. O nível de significância estatístico adotado foi de $\mathrm{p}<0,05$. Este estudo foi aprovado pelos Comitês de Ética em Pesquisa em Seres Humanos da Universidade do Estado de Santa Catarina (CEPSH/UDESC) e da Maternidade Carmela Dutra sob o CAAE: 22503713.3.0000.0118. Todas as participantes assinaram o Termo de Consentimento Livre e Esclarecido, sendo livre a decisão de interromper sua participaçáo em qualquer momento do estudo.

\section{Resultados}

Entre o período de 01 de janeiro de 2009 e 31 de dezembro de 2013, 1078 pacientes foram admitidas na Maternidade Carmela Dutra para diagnóstico e/ou tratamento oncológico. Destas, 430 correspondiam a casos analíticos de neoplasia ginecológica, sendo que, 271 cumpriram os critérios de inclusão no estudo. Após a aplicação dos critérios de exclusão e da tentativa de contato telefônico sem sucesso (90 pacientes), obteve-se uma amostra composta por 64 participantes, excluindo-se também aquelas que se recusaram a realizar o exame físico (Figura 1).

Com relação às variáveis sociodemográficas, a média de idade encontrada foi de 50 anos $(\mathrm{DP} \pm 11)$, com IMC de $27,4 \mathrm{~kg} / \mathrm{m}^{2}(\mathrm{DP} \pm 6,5)$, predominantemente cor de pele branca, identificada em 47 (73,4\%), e $36(56,3 \%)$ tiveram menos de oito anos de escolaridade. Quanto ao estado civil, 33 (51,6\%) mulheres relataram viver com companheiro e $36(56,3 \%)$ eram ativas sexualmente. As características detalhadas da amostra do estudo são apresentadas na Tabela 1.

A topografia mais frequente foi de colo uterino, correspondendo a $62,5 \%$ dos casos. Somente três pacientes $(6,3 \%)$ não foram submetidas à cirurgia, sendo a principal cirurgia realizada a histerectomia, em 35 mulheres (54,7\%). Em relação aos demais tratamentos oncológicos, foi realizada teleterapia em 14 pacientes $(21,9 \%)$, braquiterapia em $17(26,6 \%)$ e quimioterapia em 18 (28,1\%). A Tabela 2 apresenta 


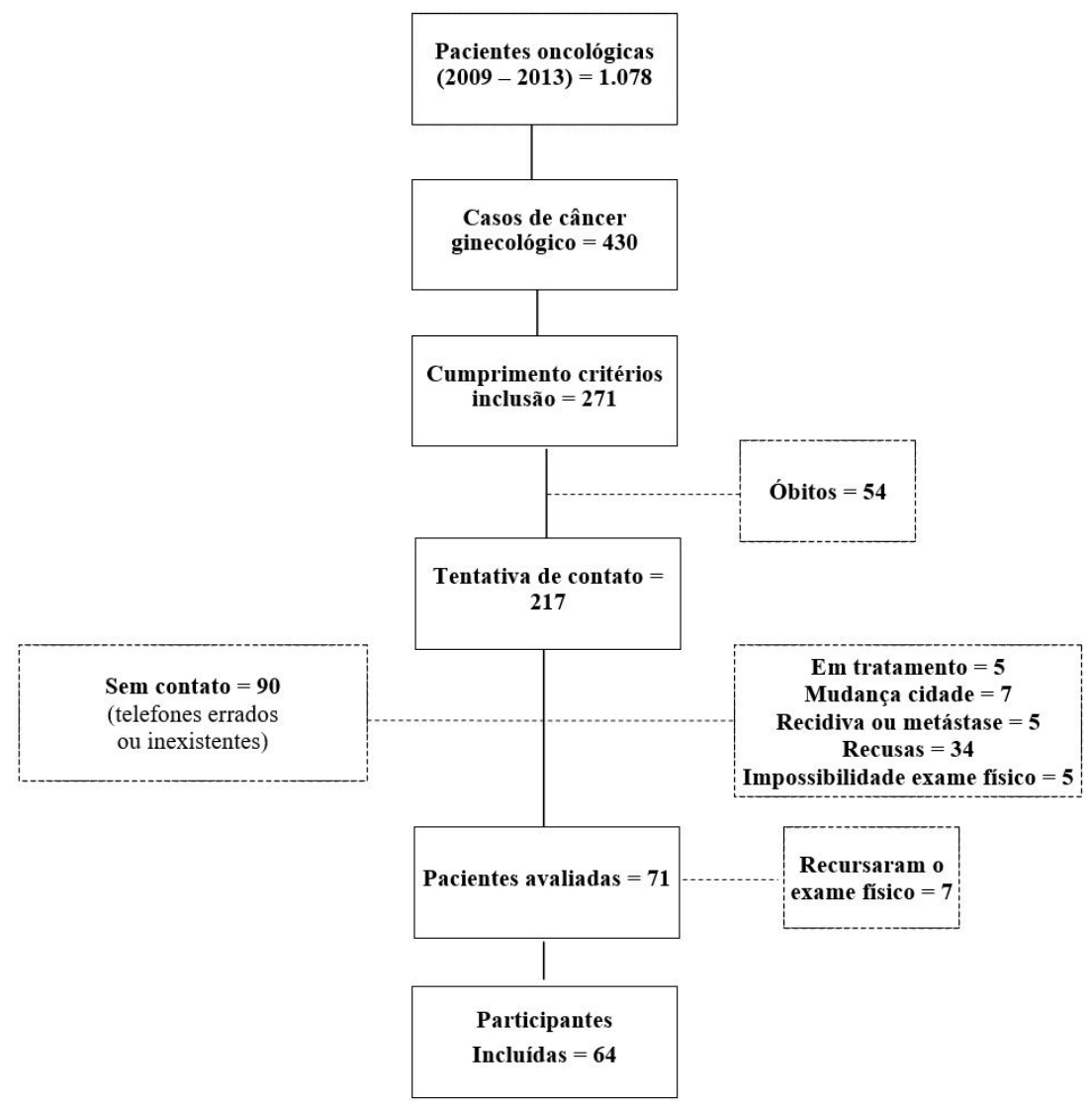

Figura 1. Organograma da seleção das participantes do estudo.

Tabela 1. Características sociodemográficas da amostra estudada.

\begin{tabular}{lc}
\hline \multicolumn{1}{c}{ Caracterização } & $\mathbf{n}(\%)$ \\
\hline Estado nutricional & $3(4,7)$ \\
$\quad$ Baixo peso & $23(35,9)$ \\
$\quad$ Peso normal & $38(59,4)$ \\
$\quad$ Sobrepeso/Obesidade & \\
Cor da pele & $47(73,4)$ \\
$\quad$ Branca & $13(20,0)$ \\
Parda & $3(4,6)$ \\
$\quad$ Preta & $1(1,5)$ \\
Amarela & \\
Escolaridade & $36(56,3)$ \\
$\quad$ Até oito anos & $28(43,8)$ \\
Acima de oito anos & \\
Estado civil & $33(51,6)$ \\
$\quad$ Com companheiro & $31(48,4)$ \\
$\quad$ Sem companheiro & \\
Menopausa & $11(17,2)$ \\
$\quad$ Não & $26(40,6)$ \\
Devido à Idade & $27(42,2)$ \\
Devido ao Tratamento & \\
Atividade sexual & $36(56,3)$ \\
$\quad$ Sim & $28(43,8)$ \\
$\quad$ Não & \\
\hline $\mathrm{n}=$ número amostral. &
\end{tabular}

as características clinicopatológicas do tumor e os tratamentos oncológicos realizados.

Embora não tenha sido avaliado o motivo da inatividade sexual (sequelas da doença/tratamento ou opção da paciente), 28 mulheres $(43,8 \%)$ tornaram-se inativas sexualmente após o tratamento oncológico. Foi detectada disfunção sexual em $18(28,1 \%)$ e dispareunia em $21(32,8 \%)$ daquelas ativas sexualmente. A presença de estenose vaginal foi verificada em $29(45,3 \%)$ casos da amostra estudada. Na avaliação da função dos músculos do assoalho pélvico, 35 mulheres (54,7\%) apresentaram contração objetiva presente. Com relação à qualidade de vida relacionada à saúde, foi obtida a média de $82,6(\mathrm{DP}=18,0)$ no escore do questionário FACT-G. A Tabela 3 mostra o percentual das complicações, função do assoalho pélvico e as medianas dos escores da qualidade de vida geral.

A comparação das médias dos escores do questionário QS-F indicou diferença significante considerando a presença ou não de estenose vaginal $(\mathrm{p}=0,02)$, e a presença ou não de dispareunia $(\mathrm{p}=0,004)$, sendo que aquelas com as complicaçôes apresentaram escores inferiores, enquanto que a presença ou ausência da contração objetiva do assoalho pélvico 
Tabela 2. Aspectos clínicos e terapêuticos do tumor da amostra estudada.

\begin{tabular}{lc}
\hline \multicolumn{1}{c}{ Caracterização } & $\mathbf{n}(\%)$ \\
\hline Topografia do tumor & \\
Colo uterino & $40(62,5)$ \\
Ovário & $13(20,3)$ \\
Endométrio & $5(7,8)$ \\
Vulva & $4(6,3)$ \\
Vagina & $2(3,1)$ \\
Estadiamento & \\
Inicial & $51(79,7)$ \\
Avançado & $9(14,1)$ \\
Sem informação & $4(6,3)$ \\
Cirurgia & \\
Sim & $61(95,3)$ \\
Não & $3(4,7)$ \\
Tipo de cirurgia & \\
Histerectomia & $35(54,7)$ \\
Conização & $17(26,6)$ \\
Outras & $9(14,1)$ \\
Não indicada & $3(4,7)$ \\
Teleterapia & \\
Sim & $14(21,9)$ \\
Não & $50(78,1)$ \\
Braquiterapia & \\
Sim & $17(26,6)$ \\
Não & $47(73,4)$ \\
Quimioterapia & \\
Sim & $18(28,1)$ \\
Não & $46(71,9)$ \\
\hline $\mathrm{n}=$ número amostral. & \\
Na &
\end{tabular}

Tabela 3. Disfunção sexual, dispareunia, estenose vaginal, contração objetiva do assoalho pélvico e qualidade de vida relacionada à saúde da amostra estudada.

\begin{tabular}{lc}
\hline \multicolumn{1}{c}{ Variáveis } & $\mathbf{n}(\%)$ \\
\hline $\begin{array}{l}\text { Disfunção sexual* } \\
\quad \text { Presente }\end{array}$ & $18(28,1)$ \\
$\quad$ Ausente & $18(28,1)$ \\
$\quad$ Inativas sexualmente & $28(43,8)$ \\
Dispareunia* & \\
$\quad$ Presente & $21(32,8)$ \\
$\quad$ Ausente & $15(23,4)$ \\
$\quad$ Inativas sexualmente & $28(43,8)$ \\
Estenose vaginal & \\
$\quad$ Presente & $29(45,3)$ \\
$\quad$ Ausente & $35(54,7)$ \\
Contração objetiva do assoalho pélvico & \\
$\quad$ Presente & $35(54,7)$ \\
$\quad$ Ausente & $29(45,3)$ \\
FACT-G & \\
$\quad$ Abaixo da mediana & $32(50,0)$ \\
$\quad$ Acima da mediana & $32(50,0)$ \\
\hline $\mathrm{n}=$ número amostral. *Apenas as pacientes ativas sexualmente \\
respondiam ao questionário QS-F.
\end{tabular}

não apresentou diferença significante na função sexual. No entanto, a presença dessas complicaçôes não gerou prejuízos significativos na qualidade de vida (FACT-G). As análises de comparação estão detalhadas na Tabela 4.

Foi encontrada associação significante entre estenose vaginal e dispareunia $(p=0,04)$. Não houve associação entre as variáveis atividade sexual e estenose vaginal e entre contração objetiva dos músculos do assoalho pélvico e estenose vaginal. Não foi verificada associação entre essas mesmas variáveis e a qualidade de vida. As análises de associação estão detalhadas na Tabela 5 .

\section{Discussão}

No presente estudo, evidenciou-se que as mulheres com estenose vaginal e dispareunia apresentaram menores escores no questionário QS-F quando comparadas àquelas sem essas complicaçóes. No entanto, a presença dessas complicaçóes não implicou em reduções significativas nos escores de qualidade de vida.

O tratamento do câncer ginecológico envolve, muitas vezes, uma abordagem multimodal, como cirurgias pélvicas extensas, radiação pélvica e/ou quimioterapia sistêmica, que podem comprometer direta ou indiretamente a anatomia, fisiologia e função dos órgãos pélvicos (FITZ et al., 2011; RUTLEDGE et al., 2014). O assoalho pélvico (AP) feminino é formado por grupos musculares e ligamentos que têm como função sustentar as vísceras abdominais e pélvicas em suas posições anatômicas (FITZ et al., 2011) em situações de aumento da pressão intra-abdominal, na respiração e na estabilização do tronco (FRANCESCHET; SACOMORI; CARDOSO, 2009). O AP controla a abertura e o fechamento da pelve promovendo e mantendo a continência urinária e fecal (FITZ et al., 2011), além de permitir a relaçáo sexual e o parto (FRANCESCHET; SACOMORI; CARDOSO, 2009). Os tratamentos oncológicos podem comprometer sua estrutura, trazendo importantes modificaçóes como a diminuição da força muscular (FITZ et al., 2011; RUTLEDGE et al., 2014), e as disfunções sexuais, entre elas a estenose vaginal, secura vaginal e dispareunia (REIS; BEJI; COSKUN, 2010; BRADFORD et al., 2015).

De fato, a presença de complicaçóes como a dispareunia e a estenose vaginal observada neste estudo aproxima-se de achados de alguns estudos longitudinais conforme Stinesen Kollberg et al. (2015) que realizaram um estudo com 616 sobreviventes 
Tabela 4. Comparação das médias dos escores do questionário QS-F e FACT-G de acordo com estenose vaginal, dispareunia e contração objetiva do assoalho pélvico.

\begin{tabular}{|c|c|c|c|c|}
\hline \multirow{2}{*}{ Variáveis } & $\mathrm{n}(\%)$ & Média \pm DP & $t^{* *}$ & p-valor \\
\hline & \multicolumn{4}{|c|}{ QS-F* } \\
\hline \multicolumn{5}{|l|}{ Estenose } \\
\hline Ausente & $19(52,8)$ & $69,37 \pm 21,6$ & 2,39 & 0,02 \\
\hline Presente & $17(47,2)$ & $49,18 \pm 28,7$ & & \\
\hline \multicolumn{5}{|l|}{ Dispareunia* } \\
\hline Ausente & $15(41,7)$ & $74,56 \pm 22,2$ & 3,09 & 0,004 \\
\hline Presente & $21(58,3)$ & $49,33 \pm 25,3$ & & \\
\hline \multicolumn{5}{|c|}{ Contração objetiva do assoalho pélvico } \\
\hline Ausente & $15(41,7)$ & $56,1 \pm 31,3$ & 0,69 & 0,5 \\
\hline \multirow{2}{*}{ Presente } & $21(58,3)$ & $62,5 \pm 23,7$ & & \\
\hline & & FACT-G & $\mathrm{Z} * * *$ & \\
\hline \multicolumn{5}{|l|}{ Estenose } \\
\hline Ausente & $35(54,7)$ & $81,2 \pm 19,6$ & $-0,57$ & 0,56 \\
\hline Presente & $29(45,3)$ & $84,3 \pm 16,2$ & & \\
\hline \multicolumn{5}{|l|}{ Dispareunia* } \\
\hline Ausente & $15(41,7)$ & $80,2 \pm 20,5$ & 0,36 & 0,37 \\
\hline Presente & $21(58,3)$ & $83,4 \pm 13,9$ & & \\
\hline \multicolumn{5}{|c|}{ Contração objetiva do assoalho pélvico } \\
\hline Ausente & $29(45,3)$ & $79,6 \pm 22,0$ & $-0,63$ & 0,53 \\
\hline Presente & $35(58,3)$ & $85,1 \pm 13,8$ & & \\
\hline
\end{tabular}

$\mathrm{n}$ = número amostral; $\mathrm{DP}$ = desvio padrão; $\mathrm{p}=$ nível de significância. *Apenas pacientes ativas sexualmente nos últimos seis meses respondiam ao questionário QS-F e a questão da dispareunia; **Teste t independente; ***Teste U de Mann-Whitney.

Tabela 5. Associação entre estenose vaginal e atividade sexual, função do assoalho pélvico e dispareunia da amostra estudada.

\begin{tabular}{|c|c|c|c|c|}
\hline \multirow{2}{*}{ Variáveis } & \multicolumn{2}{|c|}{ Estenose vaginal n (\%) } & \multirow[b]{2}{*}{$p$} & \multirow{2}{*}{$\mathbf{v}$} \\
\hline & Presente & Ausente & & \\
\hline \multicolumn{5}{|l|}{ Atividade Sexual } \\
\hline Não & $12(42,9)$ & $16(57,1)$ & 0,72 & -- \\
\hline Sim & $17(47,2)$ & $19(52,8)$ & & \\
\hline \multicolumn{5}{|l|}{ Função assoalho pélvico } \\
\hline Contração ineficiente & $16(55,2)$ & $13(44,8)$ & 0,14 & -- \\
\hline Contração eficiente & $13(37,1)$ & $22(62,9)$ & & \\
\hline \multicolumn{5}{|l|}{ Dispareunia* } \\
\hline Ausente & $4(26,7)$ & $11(73,3)$ & 0,04 & 0,34 \\
\hline Presente & $13(61,9)$ & $8(38,1)$ & & \\
\hline
\end{tabular}

$\mathrm{n}=$ número amostral; $\mathrm{p}=$ nível de significância. Teste Exato de Fisher*. v de Cramer.

de neoplasias ginecológicas, das quais $90 \%$ tinham sido tratadas com cirurgia e radioterapia adjuvante, sendo a dispareunia a principal disfunção encontrada, afetando $67 \%$ da amostra estudada. Outro estudo que comparou um grupo controle com sobreviventes ao câncer de colo uterino tratadas com radiação encontrou taxas significativas de disfunção sexual no grupo tratado, incluindo redução da dimensão vaginal (50\%), dispareunia (55\%) e falta de lubrificação (35\%) (JENSEN et al., 2003).

$\mathrm{Na}$ amostra estudada, as mulheres com estenose vaginal e dispareunia apresentaram escores inferiores no QS-F quando comparadas àquelas sem essas complicações. Um baixo nível de satisfação na função sexual de sobreviventes ao câncer ginecológico também foi relatado por Sekse, Hufthammer e Vika (2017), atribuído à alta porcentagem de relato de dor ou desconforto durante o intercurso sexual, levando a um impacto negativo na função sexual ao longo do tempo.

Alguns estudos investigaram a qualidade de vida $(Q V)$ e a disfunção sexual em pacientes após o tratamento oncológico e apontaram que, as terapias utilizadas prejudicaram a imagem corporal e levaram a um impacto negativo na sexualidade dessas pacientes (BJELIC-RADISIC et al., 2012; FLEMING et al., 
2016). Zhou et al. (2016) investigaram a qualidade de vida de 140 sobreviventes ao câncer de colo uterino, identificando uma baixa qualidade de vida relacionada a um declínio da função sexual. Outro estudo avaliou a QV de sobreviventes ao câncer ginecológico após a realização de radioterapia, mostrando que após três anos do término do tratamento a dor, dispareunia e redução do interesse sexual foram as variáveis que mais interferiram negativamente nos domínios físicos, psicológicos, sociais, impactando em piora da QV geral (VAZ et al., 2011b).

No entanto, a presença de estenose vaginal e dispareunia, não pareceu interferir negativamente na qualidade de vida relacionada à saúde no presente estudo. Hipotetiza-se que pouco se tem falado sobre os problemas da saúde sexual com as sobreviventes ao câncer ginecológico. Muitos profissionais da saúde acreditam que os problemas de saúde sexual se tornam menos importantes para essas mulheres por se tratarem de sobreviventes mais velhas (HUFFMAN et al., 2016). Além disso, as sobreviventes sofrem por falta de informaçôes, orientaçôes e constrangimento em falar sobre as questôes sexuais (REIS; BEJI; COSKUN, 2010), induzindo à conformidade e aceitação das disfunçōes do assoalho pélvico e entendendo como parte do tratamento oncológico a qual elas terão que conviver diariamente.

A estenose vaginal e a dispareunia estiveram associadas no presente estudo, conforme o esperado, pois os danos causados pelo tratamento oncológico diretamente na mucosa vaginal, nos tecidos conjuntivos e nos pequenos vasos sanguíneos podem gerar diminuição do aporte sanguíneo com subsequente hipóxia, inflamação e morte celular (SILVA et al., 2010; INTERNATIONAL..., 2012). Ainda, a formação de aderências e fibrose circunferencial prejudica a lubrificação e elasticidade da mucosa vaginal, desencadeando um quadro de dispareunia durante as tentativas sexuais ou de penetração vaginal (SILVA et al., 2010; BRAND; DO; STENLAKE, 2012; YE et al., 2014). A dispareunia, quando recorrente, pode levar à ansiedade e medo da dor durante o intercurso sexual, que por sua vez geram ainda mais tensão nos músculos do assoalho pélvico durante a tentativa de penetração vaginal (HUFFMAN et al., 2016), em um ciclo de dor-tensão-dor.

Importante destacar que cerca de $44 \%$ das participantes do estudo tornaram-se inativas sexualmente após o tratamento oncológico, ressaltando que essas mulheres inativas não responderam o questionário de função sexual. Bradford et al. (2015) observaram uma taxa de inatividade sexual de $65,8 \%$ em um estudo que avaliou a atividade sexual e a função sexual de 243 sobreviventes ao câncer ginecológico, e relataram como principais preditores de inatividade, a ausência do parceiro, o estágio da doença e o tipo de tratamento realizado. Apesar do conhecimento de tais preditores, no presente estudo náo foi avaliado o motivo da inatividade sexual das participantes.

Alguns estudos sugerem que a manutenção da atividade sexual regular em mulheres ativas sexualmente é um fator preventivo para o surgimento da estenose vaginal (LANCASTER, 2004; CARTER; GOLDFRANK; SCHOVER, 2011; PESSI et al., 2016; HUFFMAN et al., 2016). Pessi et al. (2016) realizaram um estudo descritivo utilizando como intervenção orientaçóes para a prevenção da estenose vaginal pós-braquiterapia em sobreviventes ao câncer de colo uterino, abordando a manutenção das relaçóes sexuais, de duas a três vezes por semana para as pacientes ativas sexualmente, com associação de uma prótese peniana nos casos em que a frequência das relaçôes fosse inferior à indicada. Seus resultados evidenciaram que as orientaçóes contribuíram para a redução do medo e dor durante as relaçóes sexuais, com a consequente prevençáo da estenose vaginal e melhora das relaçóes sexuais das sobreviventes com seus parceiros (PESSI et al., 2016). No entanto, não foi encontrada associação significante entre a atividade sexual e a estenose vaginal no presente estudo, indicando que mesmo as mulheres ativas sexualmente tinham estenose vaginal. No mesmo sentido, o estudo prospectivo de Decruze, Guthrie e Magnani (1999) evidenciou que 57\% de 70 mulheres sexualmente ativas apresentaram estenose vaginal um ano após o tratamento com braquiterapia, contrariando a hipótese de que a atividade sexual regular previne a estenose vaginal.

Embora não tenha sido encontrada diferença significante na função sexual entre o grupo com e sem contração objetiva do assoalho pélvico, e nem associação entre a contração dos músculos do assoalho pélvico e a estenose vaginal neste estudo, a literatura aponta que as cirurgias realizadas no tratamento contra o câncer podem alterar a anatomia pélvica, assim como modificar a inervação do assoalho pélvico, interferindo nas relaçôes sexuais (JACKSON; NAIK, 2006; ROS; ESPUNA, 2013), principalmente no mecanismo de excitação, levando a uma perda de lubrificação e sensação (JACKSON; NAIK, 2006). Um ensaio clínico randomizado demonstrou que o treinamento muscular específico intensivo melhorou a função do AP e sexual em sobreviventes ao câncer ginecológico, principalmente da contração dos músculos cavernosos, que são os principais músculos que se contraem durante a relação sexual (YANG et al., 2012). 
As sobreviventes ao câncer ginecológico enfrentam diversas barreiras no que se refere à identificação e tratamento das disfunçóes do assoalho pélvico, iniciando com a dificuldade de comunicação entre paciente e profissional da saúde (WHICKER et al., 2017). Programas multidisciplinares foram desenvolvidos buscando um plano de ação que vise a prevenir e tratar essas disfunçóes nas sobreviventes, fornecendo informaçôes e estratégias que melhoram os sintomas vaginais e proporcionam confiança em relação à saúde sexual (CARTER et al., 2017). A fisioterapia tem um papel importante no desenvolvimento de programas de reabilitação do assoalho pélvico que melhoram significativamente a função dos músculos do assoalho pélvico e a função sexual (YANG et al., 2012; CARTER et al., 2017). Algumas técnicas fisioterapêuticas de treinamento muscular, alongamento e massagem dos músculos envolvidos são estratégias promissoras que podem beneficiar as sobreviventes, refletindo na melhora da função sexual e de alguns domínios da qualidade de vida, entre eles o emocional e social (CARTER et al., 2017; WHICKER et al., 2017).

Como possíveis limitaçôes deste estudo, destaca-se a avaliação apenas subjetiva do assoalho pélvico que pode ter influenciado nos resultados encontrados. Além disso, a ausência de uma questão específica para avaliar o motivo da inatividade sexual impediu a identificaçáo de sequelas da doença/tratamento ou se a causa era opção da paciente. Dessa forma, deixou-se uma lacuna em aberto sobre os motivos da inatividade sexual nessas sobreviventes, assim como as possíveis relaçôes com as disfunções sexuais, doença e tratamento.

\section{Conclusão}

Os resultados evidenciaram que a estenose vaginal e dispareunia, quando presentes, reduziram os escores do questionário QS-F, indicando prejuízo na função sexual, sem impactar negativamente na qualidade de vida relacionada à saúde. A contração do assoalho pélvico não mostrou interferir na função sexual e na qualidade de vida das sobreviventes. Observou-se, ainda, associação entre estenose vaginal e dispareunia, porém a manutenção de atividade sexual e a contração do assoalho pélvico não mostraram ter relação com a estenose vaginal. Portanto, sugere-se que novos estudos sejam direcionados à abordagem fisioterapêutica da estenose vaginal em sobreviventes ao câncer ginecológico, com intervençôes individualizadas, e não somente baseadas em programas educacionais ou orientaçôes de manutenção de vida sexual ativa.

\section{Referências}

ABDO, C. Elaboração e validação do quociente sexual: versão feminina, uma escala para avaliar a função sexual da mulher. Revista Brasileira de Medicina, Rio de Janeiro, v. 63, n. 9, p. 477-482, 2006.

BJELIC-RADISIC, V. et al. Quality of life characteristics inpatients with cervical cancer. European Journal of Cancer, Oxford, v. 48, n. 16, p. 3009-3018, 2012. http://dx.doi. org/10.1016/j.ejca.2012.05.011. PMid:22683166.

BOBER, S. L. et al. Improvement in sexual function after ovarian cancer: effects of sexual therapy and rehabilitation after treatment for ovarian cancer. Cancer, New York, v. 124, n. 1, p. 176-182, 2018. http://dx.doi.org/10.1002/ cncr.30976. PMid:28881456.

BRADFORD, A. et al. Assessment of sexual activity and dysfunction in medically underserved women with gynecologic cancers. Gynecologic Oncology, New York, v. 139, n. 1, p. 134-140, 2015. http://dx.doi.org/10.1016/j. ygyno.2015.08.019. PMid:26325527.

BRAND, A. H.; DO, V.; STENLAKE, A. Can an educational intervention improve compliance with vaginal dilator use in patients treated with radiation for a gynecological malignancy? International Journal of Gynecological Cancer, Cambridge, v. 22, n. 5, p. 897-904, 2012. http://dx.doi. org/10.1097/IGC.0b013e31824d7243. PMid:22552831.

BRETSCHNEIDER, C. E. et al. Perioperative sexual interest in women with suspected gynecologic malignancies. Gynecologic Oncology, New York, v. 146, n. 1, p. 109-113, 2017. http://dx.doi.org/10.1016/j.ygyno.2017.04.001. PMid:28410756.

CARTER, J. et al. Vaginal and sexual health treatment strategies within a female sexual medicine program for cancer patients and survivors. Journal of Cancer Survivorship, New York, v. 11, n. 2, p. 274-283, 2017. http://dx.doi. org/10.1007/s11764-016-0585-9. PMid:27868156.

CARTER, J.; GOLDFRANK, D.; SCHOVER, L. R. Simple strategies for vaginal health promotion in cancer survivors. The Journal of Sexual Medicine, Malden, v. 8, n. 2, p. 549-559, 2011. http://dx.doi.org/10.1111/j.17436109.2010.01988.x. PMid:20722792.

DECRUZE, S. B.; GUTHRIE, D.; MAGNANI, R. Prevention of vaginal stenosis in patients following vaginal brachytherapy. Clinical Oncoogyl, London, v. 11, n. 1, p. 46-48, 1999. http://dx.doi.org/10.1053/clon.1999.9008. PMid:10194586.

FACIT. Questionnaires. Florida, 2015. Disponível em: <http://www.facit.org/FACITOrg/Questionnaires>. Acesso em: 11 jul. 2018

FITZ, F. F. et al. Impacto do tratamento do câncer de colo uterino no assoalho pélvico. Revista Feminina, Rio de Janeiro, v. 39, n. 8, p. 387-393, 2011.

FLAY, L. D.; MATTHEWS, J. H. The effects of radiotherapy and surgery on the sexual function of women treated for cervical cancer. International Journal of Radiation Oncology 
Biology Physics, Elmsford, v. 31, n. 2, p. 399-404, 1995. http://dx.doi.org/10.1016/0360-3016(94)E0139-B. PMid:7836095.

FLEMING, N. D. et al. Quality of life after radical trachelectomy for early-stage cervical cancer: a 5-year prospective evaluation. Gynecologic Oncology, New York, v. 143, n. 3, p. 596-603, 2016. http://dx.doi.org/10.1016/j. ygyno.2016.10.012. PMid:27742473.

FRANCESCHET, J.; SACOMORI, C.; CARDOSO, F. L. Força dos músculos do assoalho pélvico e função sexual em gestantes. Revista Brasileira de Fisioterapia, São Carlos, v. 13, n. 5, p. 383-389, 2009. http://dx.doi.org/10.1590/ S1413-35552009005000054.

HUFFMAN, L. B. et al. Maintaining sexual health throughout gynecologic cancer survivorship: a comprehensive review and clinical guide. Gynecologic Oncology, New York, v. 140, n. 2, p. 359-368, 2016. http://dx.doi.org/10.1016/j. ygyno.2015.11.010. PMid:26556768.

INSTITUTO NACIONAL DO CÂNCER - INCA. Estimativa 2016: incidência de câncer no Brasil. Rio de Janeiro, 2015.

INTERNATIONAL CLINICAL GUIDELINE GROUP. International guidelines on vaginal dilation after pelvic radiotherapy. Woodstock: Owen Mumford, 2012. Disponível em: <www.ncsi.org.uk/wp-content/uploads/ Inter-Best-Practice-Guide-Vaginal-Dilators-July-2012. pdf>. Acesso em: 30 out. 2018.

JACKSON, K. S.; NAIK, R. Pelvic floor dysfunction and radical hysterectomy. International Journal of Gynecological Cancer, Cambridge, v. 16, n. 1, p. 354-363, 2006. http://dx.doi.org/10.1111/j.1525-1438.2006.00347.x. PMid:16445658.

JENSEN, P. T. et al. Longitudinal study of sexual function and vaginal changes after radiotherapy for cervical cancer. International Journal of Radiation Oncology Biology Physics, Elmsford, v. 56, n. 4, p. 937-949, 2003. http://dx.doi. org/10.1016/S0360-3016(03)00362-6. PMid:12829128.

LANCASTER, L. Preventing vaginal stenosis after brachytherapy for gynaecological cancer: an overview of Australian practices. European Journal of Oncology Nursing, Edinburgh, v. 8, n. 1, p. 30-39, 2004. http://dx.doi. org/10.1016/S1462-3889(03)00059-0. PMid:15003742.

ORTIZ, O. et al. Valoración dinámica de la disfuncion perineal em la mujer: propuesta de classificación. Obstetricia y Ginecología Latino-americanas, Buenos Aires,v. 1, n. 2, p. 7-9, 1994.

PESSI, M. R. et al. Prevention of vaginal stenosis after brachytherapy: nursing interention. Journal of Nursing UFPE On Line, Recife, v. 10, n. 9, p. 3495-3502, 2016.

REIS, N.; BEJI, N. K.; COSKUN, A. Quality of life and sexual functioning in gynecological cancer patients: results from quantitative and qualitative data. European Journal of Oncology Nursing, Edinburgh, v. 14, n. 2, p. 137-146, 2010. http://dx.doi.org/10.1016/j.ejon.2009.09.004. PMid:19836305.
ROS, C.; ESPUNA, M. Impact of cervical cancer treatment on micturition and sexual function. Actas Urológicas Españolas, Madrid, v. 37, n. 1, p. 40-46, 2013. http:// dx.doi.org/10.1016/j.acuroe.2012.03.015. PMid:22728020.

RUTLEDGE, T. L. et al. A pilot randomized control trial to evaluate pelvic floor muscle training for urinary incontinence among gynecologic cancer survivors. Gynecologic Oncology, New York, v. 132, n. 1, p. 154-158, 2014. http://dx.doi.org/10.1016/j.ygyno.2013.10.024. PMid:24183730

RUTLEDGE, T. L. et al. Pelvic floor disorders and sexual function in gynecologic cancer survivors: a cohort study. American Journal of Obstetrics \& Gynecology, St. Louis, v. 203, n. 5, p. 514.e1-514.e7, 2010. http://dx.doi.org/10.1016/j. ajog.2010.08.004. PMid:20869691.

SEKSE, R. J.; HUFTHAMMER, K. O.; VIKA, E. M. Sexual activity and functioning in women treated for gynaecological cancers. Journal of Clinical Nursing, Oxford, v. 26, n. 3-4, p. 400-410, 2017. http://dx.doi. org/10.1111/jocn.13407. PMid:27239803.

SILVA, M. et al. Métodos avaliativos para estenose vaginal pós-radioterapia. Revista Brasileira de Cancerologia, Rio de Janeiro, v. 56, n. 1, p. 71-83, 2010.

STINESEN KOLLBERG, K. et al. Reduced vaginal elasticity, reduced lubrication, and deep and superficial dyspareunia in irradiated gynecological cancer survivors. Acta Oncologica, Stockholm, v. 54, n. 5, p. 772-779, 2015. http://dx.doi.org/10.3109/0284186X.2014.1001036. PMid:25761090.

VAZ, A. F. et al. Quality of life and adverse events after radiotherapy in gynecologic cancer survivors: a cohort study. Archives of Gynecology and Obstetrics, München, v. 284, n. 6, p. 1523-1531, 2011a. http://dx.doi.org/10.1007/ s00404-011-1886-x. PMid:21442259.

VAZ, A. F. et al. Quality of life and menopausal and sexual symptoms in gynecologic cancer survivors: a cohort study. Menopause, New York, v. 18, n. 6, p. 662-669, 2011 b. http://dx.doi.org/10.1097/gme.0b013e3181ffde7f. PMid:21471827.

WEBSTER, K.; CELLA, D.; YOST, K. The Functional Assessment of Chronic Illness Therapy (FACIT) measurement system: properties, applications, and interpretation. Health and Quality of Life Outcomes, London, v. 1, n. 1, p. 1-7, 2003. http://dx.doi.org/10.1186/1477-7525-1-1. PMid:14678568

WESTIN, S. N. et al. Survivors of gynecologic malignancies: impact of treatment on health and well-being. Journal of Cancer Survivorship, New York, v. 10, n. 2, p. 261-270, 2016. http://dx.doi.org/10.1007/s11764-015-0472-9. PMid:26245979.

WHICKER, M. et al. Management of sexuality, intimacy, and menopause symptoms in patients with ovarian cancer. American Journal of Obstetrics \& Gynecology, St. Louis, v. 217, n. 4, p. 395-403, 2017. http://dx.doi.org/10.1016/j. ajog.2017.04.012. PMid:28411144. 
WORLD HEALTH ORGANIZATION - WHO. Physical status: the use and interpretation of anthropometry. Geneva, 1995. (WHO Technical Report Series). Disponível em: <http://apps.who.int/iris/bitstream/10665/37003/1/ WHO_TRS_854.pdf>. Acesso em: 27 abr. 2018.

YANG, E. J. et al. Effect of a pelvic floor muscle training program on gynecologic cancer survivors with pelvic floor dysfunction: a randomized controlled trial. Gynecologic Oncology, New York, v. 125, n. 3, p. 705-711, 2012. http:// dx.doi.org/10.1016/j.ygyno.2012.03.045. PMid:22472463.

YE, S. et al. A systematic review of quality of life and sexual function of patients with cervical cancer after treatment. International Journal of Gynecological Cancer, Cambridge, v.
24, n. 7, p. 1146-1157, 2014. http://dx.doi.org/10.1097/ IGC.0000000000000207. PMid:25033255.

YOSHIDA, K. et al. Actuarial assessment and predisposing factor analysis for late vaginal stenosis after high-dose-rate brachytherapy in patients with previously untreated cervical cancer: role of vaginal pallor reaction. Journal Gynecologic Oncology, Korea, v. 16, n. 3, p. 1-6, 2015.

ZHOU, W. et al. Survey of cervical cancer survivors regarding quality of life and sexual function. Journal of Cancer Research and Therapeutics, Mumbai, v. 12, n. 2, p. 938-944, 2016. http://dx.doi.org/10.4103/09731482.175427. PMid:27461678.

\section{Contribuição dos Autores}

Clarissa Medeiros da Luz trabalhou na concepção do estudo, revisão crítica e redação da versão a ser publicada. Francielle Conceiçáo Nascimento realizou a pesquisa, análise e interpretação dos dados, assim como a redação do artigo. Julia Deitos auxiliou na pesquisa e metodologia, além da redação do artigo. Todos os autores aprovaram a versão final do texto. 